\title{
TEMPLATE MATCHING TO SUPPORT EARTH OBSERVATION BASED REFUGEE CAMP ANALYSIS IN OBIA WORKFLOWS - CREATION AND EVALUATION OF A DWELLING TEMPLATE LIBRARY FOR IMPROVING DWELLING EXTRACTION WITHIN AN OBJECT-BASED FRAMEWORK
}

\author{
P. Krafft ${ }^{\mathrm{a}}$, D. Tiede ${ }^{\mathrm{a}}$, P. Füreder ${ }^{\mathrm{a}}$ \\ a Department of Geoinformatics - Z_GIS, University of Salzburg, Austria - pascal.krafft@stud.sbg.ac.at, (dirk.tiede, \\ petra.fuereder)@sbg.ac.at
}

\begin{abstract}
KEY WORDS: Object-based image analysis (OBIA), Template matching, Object detection, dwelling extraction, refugee and IDP camps, VHR data
\end{abstract}

\begin{abstract}
:
Accurate and reliable information about the situation in refugee or internally displaced person camps is very important for planning any kind of help like health care, infrastructure or vaccination campaigns. The number and spatial distribution of single dwellings extracted semi-automatically from very high resolution (VHR) satellite imagery as an indicator for population estimations can provide such important information. The accuracy of the extracted dwellings can vary quite a lot depending on various factors. To enhance established single dwelling extraction approaches, we have tested the integration of stratified template matching methods in objectbased image analysis (OBIA) workflows. The created template library aims to be generally applicable in similar conditions. Compared to pre-existing OBIA classifications, the approach could increase in average the producer's accuracy by $12 \%$ and also slightly increase the user's accuracy. These results show that the stratified integration of template matching approaches in OBIA workflows is a possibility to further improve results of semi-automated dwelling extraction, especially in complex situations.
\end{abstract}

\section{INRODUCTION}

The number of refugees, asylum-seekers and internally displaced persons (IDP) again hits an all-time high. By the end of 2015, more than 65 million people were forcibly displaced (UNHCR, 2016) caused by natural disasters, changing environmental conditions and violent conflicts, which constitute the main reasons for displacement. Refugee camps and temporary settlements provide refuge for most of these people (Füreder et al., 2015; UNHCR, 2015). Accurate, reliable and up-to-date information about the situation in refugee and IDP camps like population estimations is very important for planning any kind of help like health care, infrastructure or vaccination campaigns. Camp management is performed typically by humanitarian relief organizations. Often it is impossible to gather such information by field assessments, because of security reasons provoked by conflict situations. Furthermore, there are sometimes incorrect information provided by stakeholders, which try e.g. to increase the number of refugees for various reasons or political interests are leading to reduced numbers of reported IDPs. IDPs have not crossed an international recognized border consequently they stay inside their home countries. Therefore the protection remains legally by their own government making assistance, such as regulated camp management, often very difficult or it is not allowed at all. The number and spatial distribution of single dwellings extracted from very high-resolution (VHR) satellite imagery can provide important information in such situations (Grundy et al., 2012; Spröhnle et al., 2014; Tiede et al., 2013). Nevertheless the accuracy of the extracted dwellings can vary quite a lot depending on various factors, such as the contrast of dwelling types to surroundings, weather situation, but also the dwelling density (Spröhnle et al., 2014). A specific limitation of existing objected-based workflows is related to the initial segmentation of the image. This process creates segments for image areas with usually similar pixel values, which can lead to under-segmentation in the case of similar spectral reflectance of the dwellings and their surroundings (e.g. under dusty conditions). On the other hand dwellings and their cast shadows can help in such situations to still distinguish dwellings from the non-elevated surroundings, but also in this case a proper segmentation of the dwelling and the shadow area is needed. Figure 1 shows an initial image segmentation where the shadow of the left dwelling is not a clearly delineated segment, which makes it hard to use the shadow as a clear identifier for a dwelling in an automated process as it is done by a visual image interpretation in the classification process.

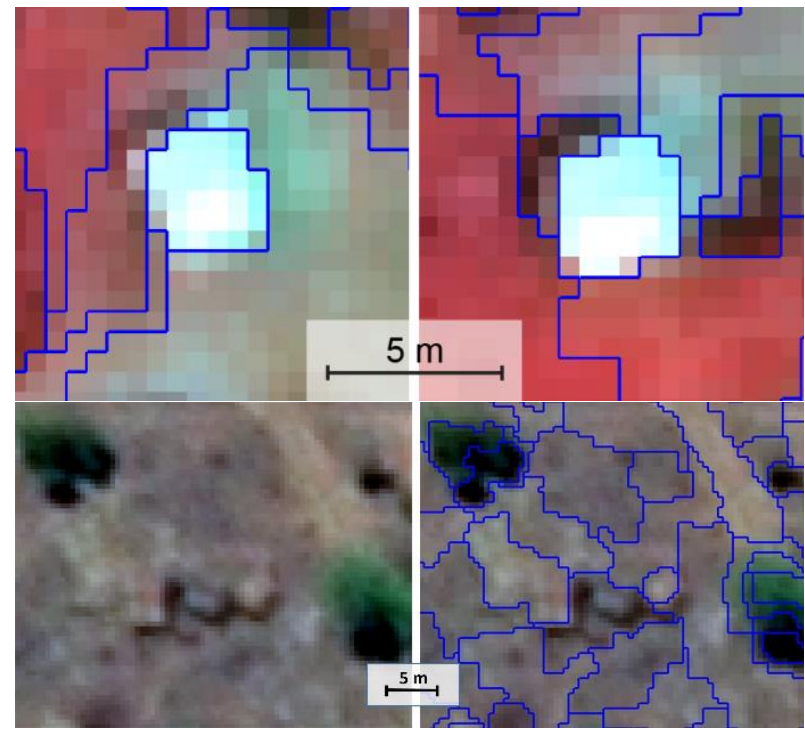

Figure 1. QuickBird 2 subsets of the Yida camp area (acquisition date: 12.10.2012, band combination NIR-R-G) showing an initial image segmentation example of two bright dwellings and the cast shadows (above). Below an example of dark dwellings which show very low contrast to the surrounding, the cast shadow is the only indicator for the presence of dwellings. Segmentation based on homogeneity usually fails in extracting meaningful objects (Yida camp, 03.04.2013, WorldView-2, R-G-B) 
To enhance single object detection in such difficult situations, we have tested the integration of stratified template matching methods in object-based image analysis workflows. In the templates generation process the object to be detected and the surroundings can be taken into account, which is in our case a dwelling and its cast shadow. Thus, matches include information regarding the orientation and can be used in the analyses to reduce false positive alarms.

The concept of template matching can be summarized as the process of comparing patterns with regard to their similarity enabling single objected detection. Although template-matching methods are not new, there is still little research on combining them with OBIA methods in one workflow. The combination of the two approaches has the aim to tackle typical problems of template matching approaches, like e.g. a high number of false positives in complex images by focusing on relevant parts of the image only (e.g. certain parts of refugee camps or certain spectrally relevant objects). The main assumption is, that the incorporation of the shadow effect of dwellings can improve the dwelling detection rate. A visual study of different refugee camps revealed that the same dwelling types often occur (bright, blue, and dark/ brown dwellings) as well as large and small structures with typical geometrical properties (cf. Figure 2). The aim of the established template library is to be generally applicable in similar conditions and to be easily integrated in already existing workflows for object-based dwelling extraction.
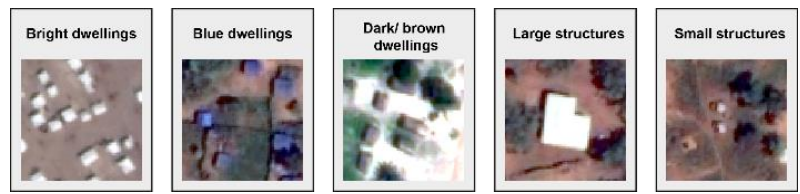

Figure 2. Typical occurring dwelling types detected in refugee and IDP camps in East Africa. Source: Füreder et al., 2015, adapted.

In order to check the accuracy of the approach it was tested on three sites of different complexities regarding dwelling extraction and the results were compared to a visual image interpretation as well as a pre-existing (independent) OBIA classifications.

\section{STUDY AREA AND DATA}

For the creation of the dwelling template library 16 VHR optical images were used. The satellite images cover parts of 10 refugee and IDP camps. These camps are distributed over Eastern Africa. The spatial distribution of the camps is presented in Figure 4. The spatial resolution ranges between $0.5 \mathrm{~m}$ and $0.6 \mathrm{~m}$ ground sample distance (pan-sharpened VHR data)

Existing semi-automated dwelling classifications were provided by the Department of Geoinformatics - Z_GIS, University of Salzburg, within an operational service for Médecins Sans Frontières (MSF) for three images (El Redis, Sudan 12.03.2015, Yida, South Sudan 12.10.2012, Yida 03.04.2013; cf. Table 1) in order to compare the outcome of the dwelling template library with existing and verified results.

\section{METHODOLOGY}

\subsection{Template matching library}

One of the objectives of this work was to create a template library for dwellings of refugee and IDP camps that can easily be integrated in already existing workflows for dwelling extraction, applicable to different kind of dwelling detection. Most of the satellite images are taken when the sun is not at the zenith of the captured region. Therefore, the library is suitable for images where objects with a certain level of height cast a shadow. The samples for the templates are taken from different camps (cf. Figure 4) to capture the variety of dwelling types at different locations, time steps and weather conditions like rainy and dry season or the effects after a sandstorm. Since most of the used images have a ground sample distance (GSD) of $0.5 \mathrm{~m} / 0.6 \mathrm{~m}$, the library is best fitting for this resolution. However, it can also be applied to other image resolutions. The applied workflow for creating a template and integration in the library is presented in Figure 3.
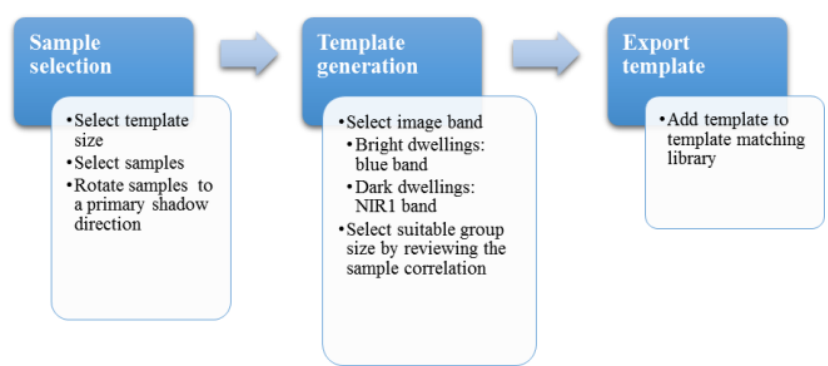

Figure 3. Generalized workflow of template generation applied in this study.

The template library is structured according to the attributes of the templates (colour intensity, dwelling shape, size and shadow direction) that allow to cover several possible combinations of a dwelling type and the cast shadow. Moreover, new dwelling templates can easily be added to the existing library enabling to customize it depending on the specific problem. The naming convention also follows this structure, making it simple for the user to find the right dwelling templates without screening all available template images. The following paragraphs describe the structure of the template library in detail.

\subsubsection{Dwelling shape}

Small objects in satellite image, such as dwellings in refugee and IDP camps look like the image were taken from bird's eye view although the VHR data is usually not collected at nadir. The building lean effect is only visible on tall objects (Baltsavias et al., 2001). Therefore, usually only the roof of a dwelling is visible and in this work the dwelling shapes are mostly referred to twodimensional geometric shapes. Dwellings in refugee camps typically have simple shapes. The predominantly simplified types are quadratic, rectangular cuboids and cones. The template library is therefore structured regarding the following three dwelling types: square, rectangle and round.

\subsubsection{Template size}

The template size is defined by the height and width of a rectangle. Templates should be big enough to cover the dwelling that is centred, the cast shadow and a bit of the surrounding area in order to include the differences of dwelling to surroundings.

\subsubsection{Dwelling brightness}

The templates are calculated in this implementation on one image band only. Hence, they are grayscale, which means that there is no distinction between colours but only between shades of grey. The template library is divided in two intensity levels: Strong intensity - bright dwellings, Weak intensity - dark dwellings. The contrast of bright dwellings compared to surrounding areas was best on the blue band. For dark dwellings, best results were 
achieved on the near infrared (NIR) band. This was detected by empirical testing and comparing the correlation of the samples of a template on the pan-sharpened multi-spectral bands (blue, green, and red and NIR).

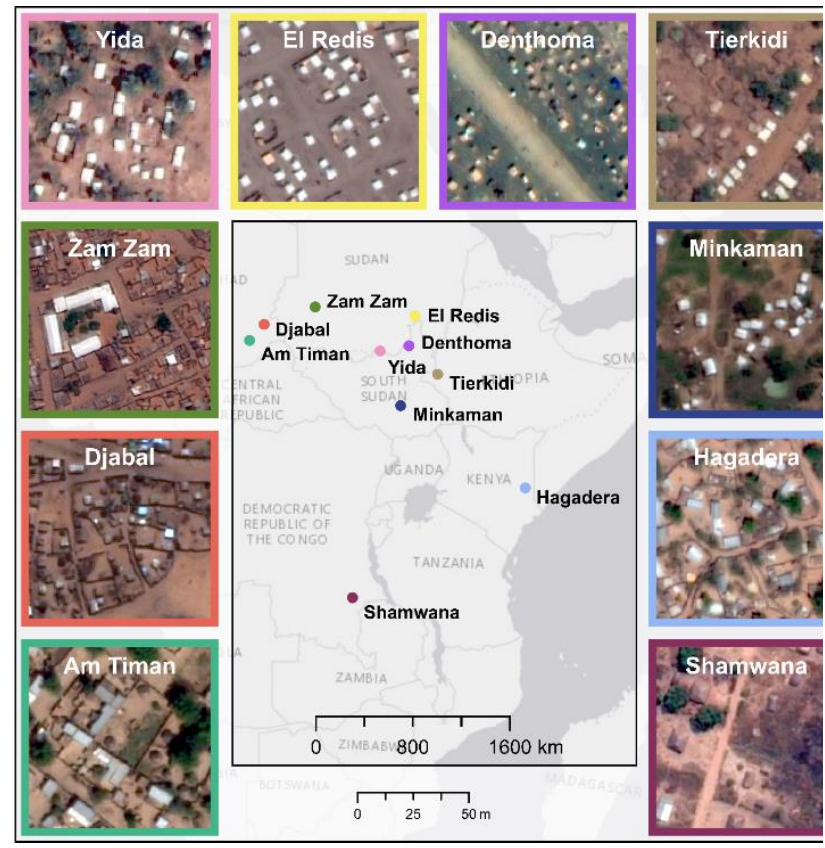

Figure 4. Spatial distribution of the refugee/IDP camps (band combination of images: R-G-B).

\begin{tabular}{|c|c|c|c|c|c|c|c|c|}
\hline & Shape & Square & Square & Rectangle & Rectangle & Rectangle & Rectangle & Round \\
\hline & Color & White & White & White & White & White & White & Grey \\
\hline & $\begin{array}{c}\text { Size } \\
{[\text { [ixel] }}\end{array}$ & $12 \times 12$ & $12 \times 12$ & $36 \times 15$ & $38 \times 15$ & $15 \times 36$ & $36 \times 15$ & $12 \times 12$ \\
\hline 裹 & Name & $\begin{array}{l}\text { Square } \\
1100 \\
\text { White } \\
12 \times 12\end{array}$ & $\begin{array}{l}\text { Square } \\
1001 \\
\text { White } \\
12 \times 12\end{array}$ & $\begin{array}{l}\text { Rectangle } \\
1000 \\
\text { White } \\
36 \times 15\end{array}$ & $\begin{array}{c}\text { Rectangle } \\
\text { 1001 } \\
\text { White } \\
38 \times 15\end{array}$ & $\begin{array}{l}\text { Rectangle } \\
1000 \\
\text { White } \\
15 \times 36\end{array}$ & $\begin{array}{l}\text { Rectangle } \\
1100 \\
\text { White } \\
36 \times 15\end{array}$ & 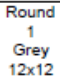 \\
\hline لِّ & $\begin{array}{l}\text { Aerial } \\
\text { view }\end{array}$ & 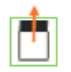 & A & & & & & \\
\hline & $\begin{array}{l}4^{\circ} \\
\text { view }\end{array}$ & 8 & $\square$ & & & & & \\
\hline \multirow{4}{*}{ 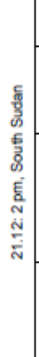 } & $\begin{array}{c}\text { size } \\
\text { [pixel] }\end{array}$ & $12 \times 12$ & $12 \times 12$ & $36 \times 15$ & $38 \times 15$ & $38 \times 15$ & $15 \times 36$ & $12 \times 12$ \\
\hline & Name & $\begin{array}{l}\text { Square } \\
1101 \\
\text { White } \\
12 \times 12 \\
\end{array}$ & $\begin{array}{l}\text { Square } \\
\text { 1000 } \\
\text { White } \\
12 \times 12\end{array}$ & $\begin{array}{c}\text { Rectangle } \\
1100 \\
\text { White } \\
36 \times 15 \\
\end{array}$ & $\begin{array}{c}\text { Rectangle } \\
\text { Wonte } \\
\text { White } \\
30 \times 15 \\
\end{array}$ & $\begin{array}{c}\text { Rectangle } \\
1001 \\
\text { White } \\
30 \times 15\end{array}$ & $\begin{array}{l}\text { Rectangle } \\
1000 \\
\text { White } \\
15 \times 30 \\
\end{array}$ & 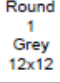 \\
\hline & $\begin{array}{l}\text { Aerial } \\
\text { view }\end{array}$ & & & & & & & \\
\hline & $\begin{array}{l}4^{\circ} \\
\text { view }\end{array}$ & $v$ & & & & & & \\
\hline
\end{tabular}

Figure 5: Structural attributes of the template library such as shadow direction, shape, type and size of dwellings.

Exemplified at predominantly occurring dwelling types on 16 different satellite images in 10 surveyed refugee and IDP camps. The shadows are simulated for the 21.12.2013 at

$12 \mathrm{am}$ and $2 \mathrm{pm}$ for the camp Yida, which is located in South Sudan. The aerial view is north oriented the $45^{\circ}$ view is west south oriented. The orange arrow shows the rotation angle and the green rectangle the (relative) size of the samples for a dwelling template.

\subsubsection{Shadow direction}

The last attribute is the shadow cast by a dwelling. It determines the rotation angle of the samples, which is highly dependent on the shape of the object. Optical VHR satellite images are typically taken at daylight. Hence, the only relevant light source is the sun. Therefore, objects cast usually only simple shadows. As mentioned above, the predominantly simplified dwelling shapes are rectangular cuboids and cones. Latter can only cast a shadow or no shadow. Rectangular cuboids have three possibilities: no shadow, a shadow on one of four sides and shadows on two sides.

The template library is built especially for dwellings showing a cast shadow. Related to the attribute shadow, objects with round layout need only one template, cubic dwellings two templates and dwellings with rectangular layout three templates because the shadow can be on one side plus on the short and the long side as well as on the long and the short side, which cannot be approached by rotating.

Summarized, the library is divided in the following main template classes (Figure 5): (a) dwellings with strong intensity, which means they appear bright on satellite images and dwellings with low intensity (structures occur dark); (b) dwellings of different shapes that can be a square, rectangle or a circle; (c) the direction of the shadow cast by the dwellings, which can be on one side or on two sides.

\subsection{Application of the template library}

The application of the templates from the library is an interactive, but straightforward process, here applied within the eCognition software environment (version 9.1, Trimble Geospatial). In order to choose the right templates from the library it is necessary to take a look at the analysed satellite images and to select the appropriate templates for potentially occurring dwellings. Within the application of the template matching algorithm, the rotation steps of the templates can be adjusted. If, for example, four rotation steps are applied, the rotation angles of the template are $0^{\circ}, 90^{\circ}, 180^{\circ}$ and $270^{\circ}$. A suitable rotation angle is always the shadow orientation of dwellings in the image. A second parameter, which can be customized, is the correlation threshold. If the correlation of the template and the image at a point is higher than the thresholds set in the configuration of the template matching process, a point ("hit") will be created. There are only points created if it is the highest values compared to the neighbouring pixels (local maximum). The resulting point layer consists typically of a lot of false positive alarms, sorting out the false alarms is essential to receive more precise results. This can be done by adjusting the correlation thresholds and filtering queries depending on the rotation angles of the templates.

\subsection{Integration of template matching in an object-based image analysis workflow}

There are three main reasons for combining template matching with objected-based image analysis methods. At first, the application of two or more different templates may result in more than one hit on the same object that means dwellings are double (or more often) counted. Second, the point layers created by applying template matching still consist of too many false positive alarms, which is reflected in a low user's accuracy, which is typical for template matching methods (Cheng and Han, 2016). Third, the templates in the library do not cover every kind 
of possible dwelling. These limitations can be overcome by stratifying the template matching with the help of OBIA methods. Multiple counting of a dwelling can be avoided by segmentation and object resizing using neighbouring information. False alarms such as trees and bushes, that cast also a shadow and therefore look quite similar compared to a dark dwelling on one image band can be sorted out easily by masking out e.g. vegetated area and by stratification of the template matching approach to the camp area only. This tasks can therefore enhance the user's accuracy significantly. Dwellings that are missed by template matching because they look not similar to one of the applied templates, but show a strong spectral distinction from the surrounding can be detected by OBIA methods. Thus, a combination of both methods can improve both, the producer's and user's accuracy. In this research, the developed template library is integrated in an OBIA workflow as outlined below (cf. Figure 5):

1) The area of interest, in this case the camp extent, is derived by an initial rough OBIA classification of dwellings and a dwelling density calculation (Tiede et al., 2013) to stratify the template matching on the camp area only and to safe computation time.

2) The template library is applied, templates and rotation of templates are selected according to a visual inspection of dwelling types and shadow directions.

3) The segmentation of the satellite image is improved by including the correlation layer of the template matching in the segmentation process.

4) Exclusion (masking) of objects that are not relevant for the analysis like vegetation and bare soil (based on spectral information) as well as elongated structures like fences or walls (based on geometrical properties). This steps helps to eliminate false positive alarms created in the template matching process.

5) Classification of the image objects based on mainly two steps: (i) classification of dwellings using the template point layer and (ii) additional classification of dwellings applying OBIA methods (objects which do not match the template matching process, but show characteristics of dwellings regarding spectral information, form and spatial embeddedness).

\section{RESULTS AND DISSCUSION}

\subsection{Results for three test sites}

The template library was applied on three images showing different levels of difficulty regarding dwelling extraction. The used camp sites are El Redis and two different points in time of the camp Yida (cf. Table 1). The provided images cover parts of the Yida camp and the complete El Redis site. In the following, results of three different methods which were applied on the three sets of images are presented. The applied approaches for dwelling detection are: (1) Template library that is based on grayscale image template matching; (2) Template library for dwelling extraction within an object-based framework. As comparison existing and user-validated OBIA classifications for the sites were available.

The output of the template matching implementations is a point file. Applying more than one template creates sometimes several hits on one dwelling. In order to merge these multiple hits to get a meaningful result, the points are buffered with a radius of $1.5 \mathrm{~m}$. Consequently, the diameter is $3 \mathrm{~m}$, which is approximately the size of a small dwelling type.

The complexity regarding dwelling extraction of the image taken from the El Redis camp acquired at 12.03.2015 can be rated as 'low'. It consists mostly of bright dwellings, which can be clearly distinguished from surrounding areas. However, some darker dwellings are harder to distinguish. The structure of the camp is very clear. There is almost no vegetation like trees and bushes within the camp area, which could be wrongly identified as a dwelling. Fences, which can look like a shadow cast by a dwelling, occur rarely. The last two facts reduce false positive alarms created by template matching and misclassification.

\begin{tabular}{|l|l|l|l|l|}
\hline Site & $\begin{array}{l}\text { Acquisition } \\
\text { date }\end{array}$ & Size [pixel] & Sensor & Complexity \\
\hline $\begin{array}{l}\text { El } \\
\text { Redis }\end{array}$ & 12.03 .2015 & $1657 \times 1658$ & WV-2 & Low \\
\hline Yida & 12.10 .2012 & $2698 \times 2337$ & QB & Average \\
\hline Yida & 03.04 .2013 & $3237 \times 2805$ & WV-2 & High \\
\hline
\end{tabular}

Table 1. Sites on which the template library is applied including information about acquisition date, size and complexity regarding dwelling extraction.

Without a differentiation between dwelling types the application of the template library only, results in 2159 detected dwellings. Integrated in an object-based framework the classified dwellings were reduced to 1542 . In comparison to these results the preexisting OBIA classification detects 1426 dwellings. Differentiated between dwelling types the pre-existing OBIA classification detects 1261 bright dwellings, 139 brown dwelling and 26 large structures. The approach of the template library within an OBIA framework extracts 1357 bright dwellings, 168 brown dwellings and 17 large structures (cf. Figure 7). All in all, the new approach combined with OBIA methods detects 116 dwellings more than the pre-existing OBIA classification in the same area.

A reason for the difference is that the pre-existing OBIA classification lacks in creating discrete segments for dwellings that are built near to each other. Thus, a segment can includes more than one object. If the area of this segment is greater than the threshold for large structures, it is classified as a large structure. This could be improved by the new approach by integrating a correlation layer in the segmentation process. In comparison to the image captured from the El Redis camp the complexity increases from 'low' to 'average' in the image from the Yida camp acquired at $12^{\text {th }}$ October 2012. It consists mostly of bright and blue dwellings, typically built with local material and covered by white or blue plastic sheets in the rainy season (UNHCR, 2013). Bright structures can be clearly distinguished from surrounding areas compared to darker structures, which are more difficult to detect. The detection rate of blue dwellings depends on the intensity and similarity of surrounding areas. The camp structure can be described as 'chaotic'. The reason for this is that the development of the camp in its initial phase was not managed by a humanitarian relief organisation (nubareports.org, 2015). Most of the dwellings are located disperse. Between these structures, trees and other vegetation like grassland and rustcoloured earthen patches can be found. This increases the complexity concerning dwelling extraction. Especially if structures are partial hidden by tree crowns or look similar to surrounding areas, the differentiation of dwellings is hampered. The image was taken in the end of the rainy season in October. Therefore, the vegetation appears intense and dwelling roofs are less dusty. Overall, that increases the contrast between dwellings and surrounding areas. Occasionally fences that appear similar to shadows cast by a dwelling, occur. Overall the template library based approach detects in this case 5447 dwellings. The combined method extracted 2913 bright dwellings, 1644 blue dwellings, 90 large structures - in total 4674 dwellings. 


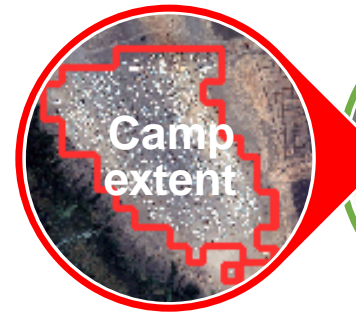

- Rough segmentation on one image band

- Classification of clear dwellings

$\rightarrow$ camp extent

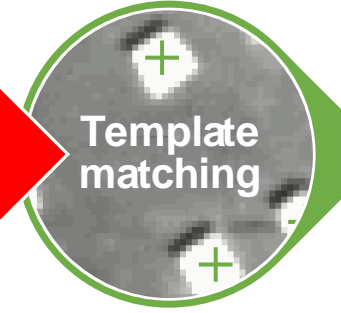

- Application of template library

- Refinement of result using correlation and rotation thresholds

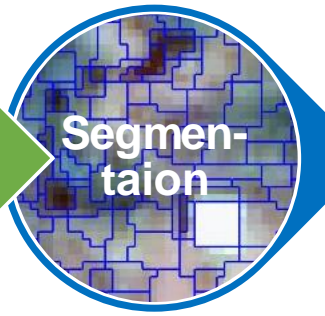

- Segmentation using various image bands, indexes and template correlation layer

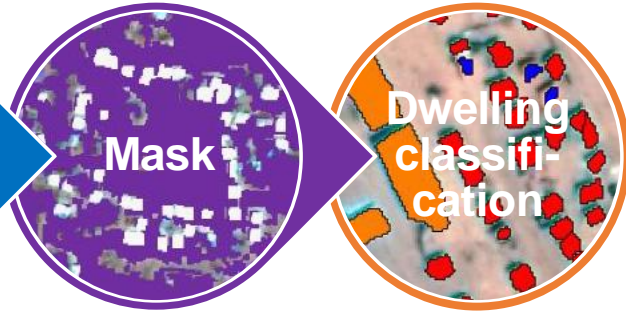

- Creation of a mask, where no dwellings can occur like:

- Vegetation mask

- Ground mask - etc.
- Classification using template points

- Classification using OBIA methods

- Refinement of classes

Figure 5. Overview of the applied combination of OBIA and template matching for dwelling extraction

In comparison, the pre-existing OBIA classification detects 3095 bright dwellings, 1268 blue dwellings and 67 large structures, which is 4430 dwellings in total (cf. Figure 7). The differences in bright and blue dwellings result from slightly different thresholds that distinguish these structures. Overall, the template matching library integrated in an object-based framework approach detects 217 dwellings more than the pre-existing OBIA classification in the same area.

The third image also covers the camp Yida and was taken six months later in early April 2013. The different sizes of the subsets are due to the different GSDs of different sensors (QuickBird with $0.6 \mathrm{~m}$ GSD and WorldView- 2 with a GSD of $0.5 \mathrm{~m}$ ). This scene was chosen in order to compare the effects and impacts of dry versus rainy season. There was only little change in terms of the camp structure, distribution of dwellings and occurrance of fences. However, this time the image is taken at the end of the dry season. The impact of this change is, that the complexity for dwelling extraction increases to 'high' because of several reasons. Dwelling roofs look dusty and are similar to the surroundings, where mainly rust-coloured earth and some trees can be found (cf. Figure 6). All in all, there is less contrast between all image objects that influences the accuracy of the detection of dwellings and classification respectively.
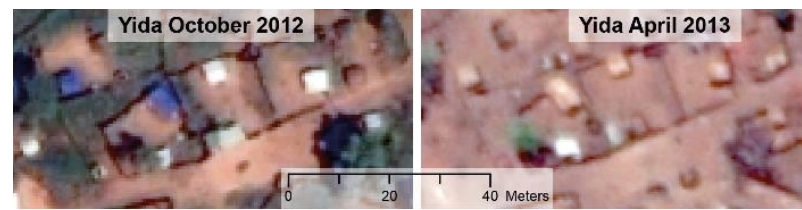

Figure 6. Comparison of the same area of the Yida camp (80m x 40m) between October 2012 (left, QuickBird) and April 2013 (right, WV-2). In the rainy season (left) dwelling show higher contrast to surroundings compared to the dry season (right).

Not differentiated between dwelling types, the template library based approach detects 4789 dwellings. The pre-existing OBIA classification detects 1665 bright, 1839 brown and 264 blue dwellings. Additionally, there are the classes large structure with 538 objects and small structure with 123 objects (in total 4429 dwellings were detected). In comparison, the template library within an object-based framework detected in total 5532 dwellings. Broken down by classes there are 2455 bright, 2277 brown and 289 blue dwellings as well as 330 large structures and 181 small structures (cf. Figure 7). Overall, 1103 additional dwellings are extracted compared to the pre-existing OBIA classification.

It should be emphasized that this time the template matching library detected fewer dwellings than the combined approach. Most likely the applied templates did not cover the whole range of occurring dwellings, especially the dusty conditions caused by dry season result in less clear differentiation between bright and dark structures which is hampering template matching using grey level based templated only.

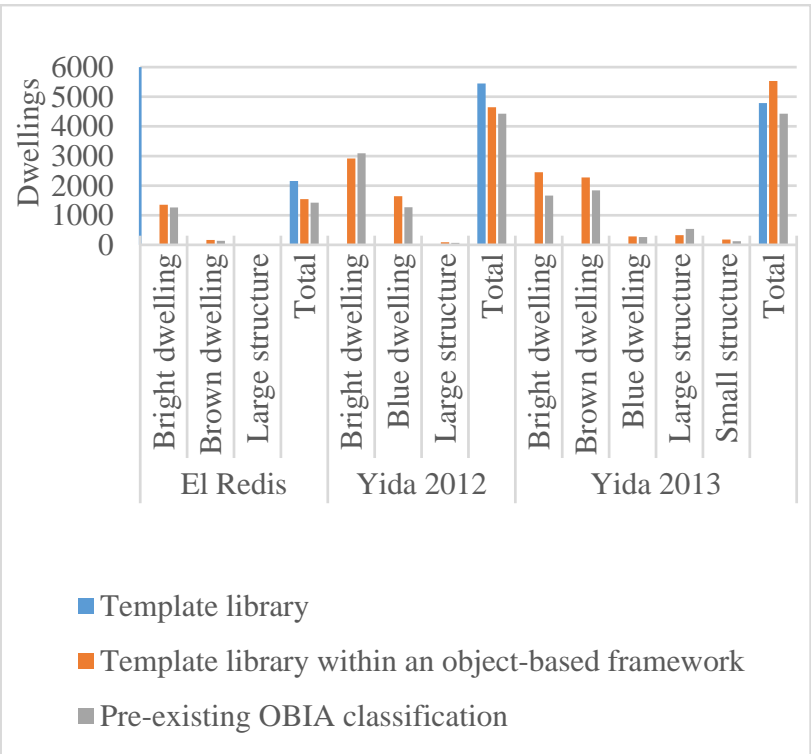

Figure 7. Number of detected dwellings for the three test sites

\subsection{Accuracy assessment}

In general the success of a method coincides with the applicability and accuracy. In order to verify latter, the results from the three test sites were compared in addition with a visual interpretation based on the satellite images. The visual interpretation was performed on randomly selected $200 \mathrm{~m}$ x $200 \mathrm{~m}$ sample areas. In the El Redis camp only two of these sample areas were created because of the small camp size. In the Yida camp five sample areas were created (cf. Figure 8). The sample areas are the same for both years (2012 and 2013), which allows a detailed comparison of the development in these camp parts. The visual interpretation includes a point file for every occurring 
dwelling in the sample areas, including attributes about the dwelling type.

\subsubsection{Accuracy assessment: not differentiated between dwelling types}

For El Redis the visual interpretation revealed 657 dwellings within the randomly selected sample areas. The template library identified 602 dwellings correctly, showing a user's accuracy of approximately $74 \%$ and a producer's accuracy of $92 \%$. Integrated template matching within an object-based framework extracted the same number of correctly extracted dwellings (same producer's accuracy), but the user accuracy increased to approximately $98 \%$ based on the reduction of false positive alarms. In comparison, the pre-existing OBIA classification extracted 542 dwellings correctly with a very high user's accuracy of $96 \%$ but a lower producer's accuracy of $83 \%$ compared to the other approaches (cf. Figure 9).
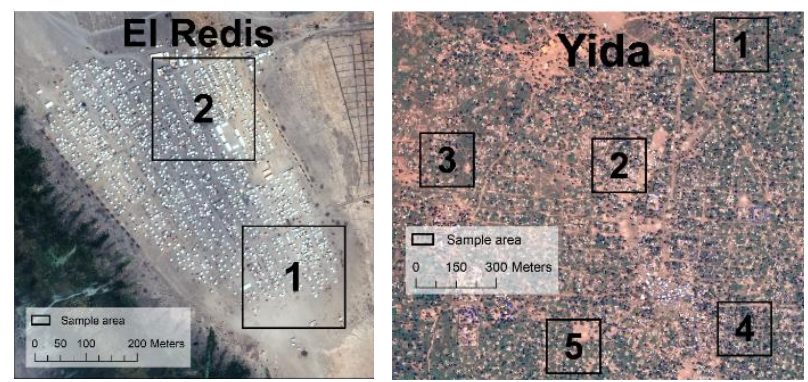

Figure 8. On the left side El Redis camp (2015, band combination R-G-B) and on the right side Yida camp (2012, band combination R-G-B) with square cells (200m x 200m) randomly selected for visual interpretation and accuracy assessment.

For the image of the Yida camp captured in October 2012, the visual interpretation detected 587 dwellings within the sample areas. Applying the template library based approach, 410 dwellings were classified correctly with a user's accuracy of 75\% and a producer's accuracy of $70 \%$. The combination with OBIA methods increased the correctly detected structures to 452 and corresponding the producer's accuracy to $77 \%$. False positive alarms are reduced strongly, which shows the enhanced user's accuracy of $93 \%$. For comparison, the pre-existing OBIA classification extracted 397 dwellings with a high user's accuracy of $90 \%$ and a producer's accuracy of $68 \%$ (cf. Figure 9 ).

For the same area, but based on the image acquired six months later, the visual interpretation revealed 504 dwellings. The template matching only detects 247 dwellings with a user's accuracy of $65 \%$ and a producer's accuracy of $49 \%$. Integrated in an object-based framework the extracted number of dwellings increased to 374 and hence also the producer's accuracy to $74 \%$. Moreover, the user's accuracy rises to $77 \%$. The pre-existing OBIA classification extracted 284 dwellings correct with a user's accuracy of $78 \%$ and a quite low producer's accuracy of $56 \%$ (cf. Figure 9).

\subsubsection{Accuracy assessment: differentiated between dwelling types}

A differentiation between dwelling types was conducted for the application of the template library within an object-based framework and for the pre-existing OBIA classifications only. Applying a template matching implementation frequently results in multiple hits per dwelling. A dark dwelling template can create a positive alarm on structures appearing dark as well as for structures which appear brighter (but with similar contrast) vice versa for a bright dwelling template. Consequently, no precise and accurate distinction of dwelling types can be conducted. Differentiated between dwelling types the template library within an object-based framework detected 598 dwellings correct in the El Redis camp test site. The user's and producer's accuracy decreases less than $1 \%$ compared to the result without the differentiation between dwelling types. Looking at the single classes the producer's accuracy is very high for bright dwellings (95\%) and large structures $(100 \%)$ but only moderate for brown dwellings $(66 \%)$. The overall accuracy reduction of the preexisting OBIA classification (537 extracted structures) is also less than $1 \%$. Important to mention are the high producer's accuracy rates for bright dwellings $(90 \%)$ and large structures $(100 \%)$ while there is a very low value for brown dwellings (33\%), which are much better detected by the integrated template matching approach. The low accuracy values for brown dwellings do not have a lot of influence on the overall accuracy of both classifications because their dwelling share is approximately $15 \%$.

For the image of the Yida camp (October 2012) the template matching combined with OBIA methods extracted 452 dwellings correctly, which is less than without a dwelling distinction. Hence, this reduces the user's and producer's accuracy (user's accuracy: 91\%, producer's accuracy: 75\%). The class-specific user's and producer's accuracy depend again highly on dwelling types (class-specific user's accuracy: $96 \%$ for bright dwellings, $81 \%$ for blue dwellings and $67 \%$ for large structures; classspecific producer's accuracy: $78 \%$ for bright dwellings, $70 \%$ for blue dwellings and $67 \%$ for large structures). As a comparison, the user's accuracy is $88 \%$ and the producer's accuracy is $66 \%$ for the pre-existing OBIA classification. The class-specific user's and producer's accuracy develop in a similar way (class-specific user's accuracy: 93\% for bright dwellings, $80 \%$ for blue dwellings and $33 \%$ for large structures; class-specific producer's accuracy: $77 \%$ for bright dwellings, $48 \%$ for blue dwellings and $67 \%$ for large structures). A particularly conspicuous aspect is that the template matching within an object-based framework detects blue dwellings a lot better than the pre-existing OBIA classification only.

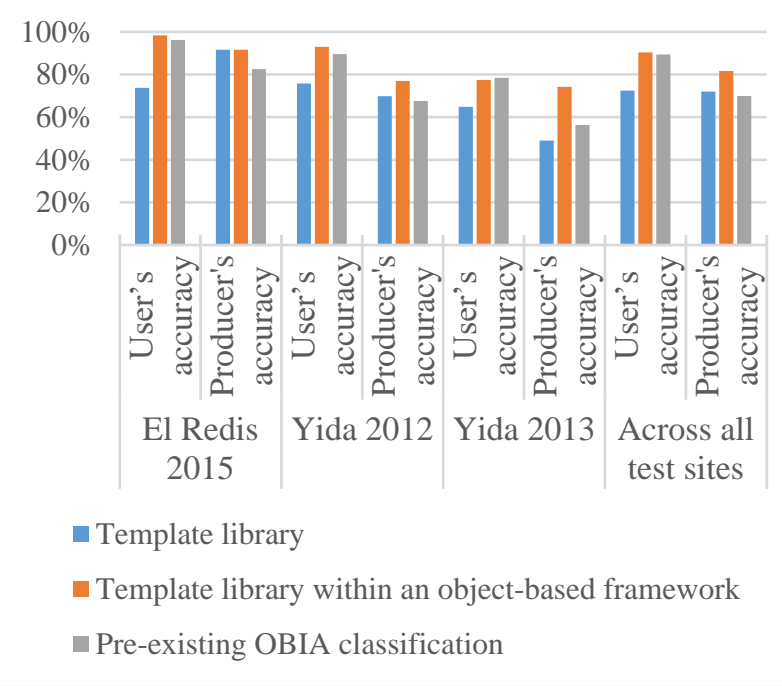

Figure 9. Accuracy assessment for randomly selected sample areas. Dwellings are not differentiated by type.

For the second image taken from the Yida camp, the combined approach detects 346 dwellings correctly (differentiated by type). The producer's accuracy drops to $68 \%$ as well as the user's accuracy to $71 \%$. A detailed look at the class-specific accuracy 
reveals again that darker structures are harder to extract (classspecific user's accuracy: $69 \%$ for bright dwellings, $76 \%$ for brown dwellings, $81 \%$ for blue dwellings, $56 \%$ for large structures and $25 \%$ for small structures; class-specific producer's accuracy: $90 \%$ for bright dwellings, 59\% for brown dwellings, $52 \%$ for blue dwellings, $59 \%$ for large structures and $100 \%$ for small structures). The pre-existing OBIA classification extracted 238 dwellings in total, with a user's accuracy of $65 \%$ and a producer's accuracy of $47 \%$. The class-specific accuracy shows that the detection rate for all classes apart from large structures is low (class-specific user's accuracy: 67\% for bright dwellings, $73 \%$ for brown dwellings, $91 \%$ for blue dwellings, 34\% for large structures and $15 \%$ for small structures; class-specific producer's accuracy: $61 \%$ for bright dwellings, $40 \%$ for brown dwellings, $44 \%$ for blue dwellings, $71 \%$ for large structures and $67 \%$ for small structures) in comparison to the template matching within an object-based framework. Figure 10 presents the accuracy differentiated by the dwelling type summarised across all test sites.

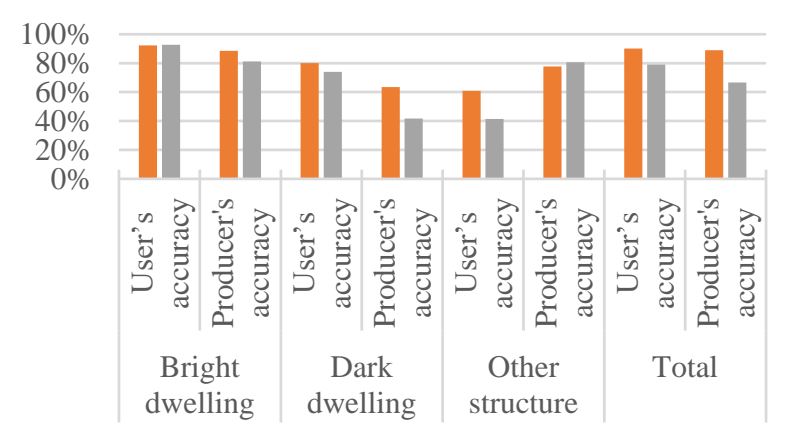

- Template library within an object-based framework

$\square$ Pre-existing OBIA classification

Figure 10. Accuracy assessment for randomly selected sample areas. Dwellings are differentiated by the type.

\section{CONCLUSION AND OUTLOOK}

The main finding of this study is that template matching is suitable for dwelling extraction in refugee and IDP camps using VHR satellite imagery, if it is stratified by OBIA means. Incorporating the shadow effect of dwellings in the templates improved the detection rate. Furthermore, the orientation of a template caused by the shadow can be used as a clear identifier for a dwelling. This is an advantage compared to conventional object-based workflows for dwelling extraction. A visual analysis of several refugee camps in East Africa revealed that there are certain dwelling types which typically occur. Based on this findings a template library for various dwelling types (template samples are taken from ten different camp sites using 16 satellite images) was established. The library is structured according to the dwelling grayscale intensity (strong: bright dwelling, weak: dark dwelling), dwelling shape (square, rectangle, circle), dwelling size (in pixel) and shadow direction (one side, two sides). The bright dwelling templates are created on the blue band and dark dwellings on the NIR band, based on empirical tests. Altogether 18 template classes were created covering typically occurring dwellings. The template matching library aims to be generally applicable in similar conditions. The tests implementations revealed, that if the template library is applied solely, it is sensitive to image areas which look similar to a template such as trees or a white spot on the ground. Consequently, false positive alarms are represented by a low user's accuracy. This drawback could be overcome by a stratified usage in OBIA workflows.

It could be shown, that the combined approach, stratifying the template matching within an initial object-based analysis, increases the average producer's accuracy by $11.7 \%$ and slightly increase the user's accuracy by $0.9 \%$ compared to pre-existing conventional OBIA classifications. This holds also true, if the accuracy assessment is further differentiated into different dwelling types.

These results show, that the integration of template matching in OBIA workflows is a possibility to further improve results of semi-automated dwelling extraction, especially in complex situations. The initial assumptions could therefore be verified: (1) Through the incorporation of the shadow effect of a dwelling in a template library, the extraction rate in difficult (e.g. low contrast, dense dwellings) situations can be improved; (2) it is possible to establish a general template matching library for dwellings to be applied in similar conditions; (3) only the combination of template matching with OBIA methods (stratification) can enhance the accuracy of dwelling extraction compared to template matching solely.

Restrictions of the used template matching implementation are (i) the missing support for multiband images; (ii) templates are not scale invariant and (iii) the rotation angle could not be set directly but only the amount of rotation numbers, which increases the computation time (redundant usage of templates) in the process.

The accuracy of grayscale template matching can further be increased, if a multiband template matching approach is used. First tests in the software Ciratefi (v.1.05) revealed quite promising results, but the combination with OBIA workflows is at the moment limited due to the lack of geo-spatial data support and reduced radiometric depth allowed per image band. For a multiband approach also the templates library needs to be extended, which could hamper the general applicability along with increasing computation time.

\section{ACKNOWLEDGEMENTS}

The research has received funding from the Austrian Research Promotion Agency (FFG) under the Austrian Space Application Programme (ASAP) within the projects EO4HumEn and EO4HumEn+ (EO-based services to support humanitarian operations: monitoring population and natural resources in refugee/IDP camps; contract no: 840081/854041).

\section{REFERENCES}

Baltsavias, E.P., Gruen, A., VanGool, L., 2001. Automatic Extraction of Man-made Objects from Aerial and Satellite Images III, Monte Verit $\{a ̀\}$. Taylor \& Francis.

Cheng, G., Han, J., 2016. A Survey on Object Detection in Optical Remote Sensing Images. ISPRS JOURNAL OF PHOTOGRAMMETRY AND REMOTE SENSING 117, $11-28$.

Füreder, P., Lang, S., Rogenhofer, E., Tiede, D., Papp, A., 2015. Monitoring Displaced People in Crisis Situations Using Multi-temporal VHR Satellite Data During Humanitarian Operations in South Sudan, in: GI_Forum 2015 Geospatial Minds for Society. pp. 391-401.

Grundy, C., Füreder, P., Siddiqui, R., Katsuva Sibongwe, D., Tiede, D., Lang, S., Checci, F., 2012. Validation of satellite imagery methods to estimate population size., in: MSF Scientific Day, 25 May 2012. London, Great Britain.

Lang, S., Tiede, D., Hölbling, D., Füreder, P., Zeil, P., 2010. Earth observation (EO)-based ex post assessment of 
internally displaced person (IDP) camp evolution and population dynamics in Zam Zam, Darfur. International Journal of Remote Sensing 31, 5709-5731.

nubareports.org, 2015. On the move again; 70,000 refugees compelled to leave camp, Nuba Reports. http://nubareports.org/on-the-move-again-70000refugees-compelled-to-leave-camp/ (08.07.2016)

Spröhnle, K., Tiede, D., Schoepfer, E., Füreder, P., Svanberg, A., Rost, T., 2014. Earth Observation-Based Dwelling Detection Approaches in a Highly Complex Refugee Camp Environment - A Comparative Study. Remote Sensing 6, 9277-9297.

Tiede, D., Füreder, P., Lang, S., Hölbling, D., Zeil, P., 2013. Automated Analysis of Satellite Imagery to provide Information Products for Humanitarian Relief Operations in Refugee Camps - from Scientific Development towards Operational Services. PFG Photogrammetrie Fernerkundung - Geoinformation 2013, 185-195.

UNHCR, 2013. UNHCR Statistical Yearbook 2012, 12th edition 172.

UNHCR, 2015. Mid-Year Trends 2015. http://www.unhcr.org/statistics/unhcrstats/56701b969/mi d-year-trends-june-2015.html (08.07.2016)

UNHCR, 2016. Global Trends. Forced displacement in 2015. http://www.unhcr.org/news/latest/2016/6/5763b65a4/glo bal-forced-displacement-hits-record-high.html (08.07.2016) 